\title{
Synergistic effect of peroxiredoxin II antisense on cisplatin- induced cell death
}

\author{
Young Do Yoo ${ }^{1,2}$, Young Min Chung ${ }^{3}$, Jong Kuk \\ Park $^{4}$, Chul Min Ahn ${ }^{5}$, Sung Kyu $\mathrm{Kim}^{5}$ and \\ Hyung Jung Kim ${ }^{5,6}$ \\ ${ }^{1}$ Korea University Cancer Institute, Korea University College of \\ Medicine, Seoul 136-705, Korea \\ ${ }^{2}$ Genomic Research Center for Lung and Breast/Ovarian Cancers, \\ Korea University College of Medicine, Seoul 136-705, Korea \\ ${ }^{3}$ Brain Korea 21 Biomedical Sciences, Korea University College of \\ Medicine, Seoul 136-705, Korea \\ ${ }^{4}$ Department of Life Science, Sogang University, Seoul 100-611, \\ Korea \\ ${ }^{5}$ Department of Internal Medicine, Yonsei University College of \\ Medicine, Seoul 135-270, Korea \\ ${ }^{6}$ Corresponding author: Tel, +82-2-3497-3316; \\ Fax, +82-2-3463-3882; E-mail, khj57@yumc.yonsei.ac.kr
}

Accepted 22 July 2002

Abbreviations: Prx II, peroxiredoxin II; Cl, combination index

\begin{abstract}
Peroxiredoxin II (Prx II) is known not only to protect cells from oxidative damage caused by hydrogen peroxide $\left(\mathrm{H}_{2} \mathrm{O}_{2}\right)$, but also to endow cancer cells with resistance to both $\mathrm{H}_{2} \mathrm{O}_{2}$ and cisplatin and to grant them radioresistance. In this study, we examined whether Prx II antisense could enhance cisplatin-induced cell death. When gastric cancer cells were transfected with various concentrations of Prx II antisense plasmid, pPrxII/AS, and then treated with the same concentrations of cisplatin, Prx II antisense enhanced cisplatininduced cell death. The combination index $(\mathrm{Cl})$ at all doses of the combination was below 1 , indicating that Prx II antisense sensitized cisplatin-induced cell death. This synergism was also observed in the cells transfected with a Prx II antisense oligomer. Our present results, therefore, suggest that Prx II antisense would be a very good sensitizer for cisplatin, and that Prx II as a target for chemosensitizers constitutes a promising avenue for future research.
\end{abstract}

Keywords: Prx II, cisplatin, chemosensitizer, antisense, multidrug resistance

\section{Introduction}

Prx II is an antioxidant enzyme that reduces $\mathrm{H}_{2} \mathrm{O}_{2}$ and other reactive oxygen species using thioredoxin as the immediate electron donor (Chae et al., 1994), and its peroxidase activity prevents cells from reactive oxygen species insult (Chae and Rhee, 1999). Prx II is also involved in the cellular signaling pathways of growth factors and tumor necrosis factor- $\alpha$, by virtue of its regulation of intracellular $\mathrm{H}_{2} \mathrm{O}_{2}$ (Kang et al., 1998; Sen, 1998). Six Prx isoforms have been identified which, based on the amino acid sequences, are generally divided into two subfamilies; groups I, II, III, and IV with two conserved cysteines and groups $\mathrm{V}$ and $\mathrm{VI}$ with one conserved cysteine (Butterfield et al., 1999). Prx I and Prx II proteins are known to be located in cytoplasm (Chae et al., 1999).

$\mathrm{Prx}$ overexpression is frequently observed in certain types of cancer tissues. Three types of Prx (I, II, and III) have been shown to be overexpressed in the case of human breast cancer, and it has been suggested that their overexpressions are related to cancer development or progression (Noh et al., 2001). The increased expression of Prx I is also detected in lung cancer, thyroid tumors and oral cancer, and is suggested to constitute a potential tumor marker (Yanagawa et al., 1999; Yanagawa et al., 2000; Chang et al., 2001). Prx II is upregulated by $\mathrm{H}_{2} \mathrm{O}_{2}$ and cisplatin treatment, and its increased expression inhibits the apoptosis induced by cisplatin, thyrotropin, serum deprivation, ceramide, or etoposide, thereby rendering tumor cells resistant to some chemotherapeutic agents (Zhang et al., 1997; Kim et al.,2000; Chung et al., 2001). We demonstrated earlier that Prx II was involved in radioresistance (Park et al., 2000). In the present study, therefore, we explored the possibility as to whether inhibition of Prx II expression with antisense or drugs would accelerate cell death induced by anticancer agents.

\section{Materials and Methods}

\section{Drug sensitivity assay}

Human gastric-carcinoma cells (SNU638), were cultured at $37^{\circ} \mathrm{C}$ under $5 \% \mathrm{CO}_{2}$ in an RPMl 1640 medium (GIBCO/BRL, Grand Island, NY), containing 10\% heatinactivated fetal bovine serum, sodium bicarbonate (2 $\mathrm{mg} / \mathrm{ml})$, penicillin (100 units $/ \mathrm{ml}$ ) and streptomycin (100 $\mu \mathrm{g} / \mathrm{ml})$. Exponentially growing SNU638 cells $\left(2 \times 10^{5}\right)$ were transferred to $60-\mathrm{mm}$ culture dishes, and the cells were then transfected with various concentrations of the antisense Prx II expression vector (pPrxll/AS) or with antisense/sense oligomers combined with a mixture of cationic liposome (GIBCO/BRL). The cells were added 
to the culture medium and then cultured for $16 \mathrm{~h}$. The process of transfection was then repeated a second time. The cells were treated with various doses of cisplatin $16 \mathrm{~h}$ after transfection. After 4 days of incubation at $37^{\circ} \mathrm{C}$, surviving cells were counted under a microscope.

\section{Determination of apoptotic cell death}

SNU638 cells $\left(1 \times 10^{6}\right)$ were transferred to $100-\mathrm{mm}$ culture dishes and the cells were transfected with 0.5 $\mu \mathrm{g} / \mathrm{ml}$ of the antisense Prx II expression vector (pPrxll/ AS) along with a mixture of $5 \mu \mathrm{g}$ of cationic liposome (GIBCO/BRL), and the process was repeated a second time. After $16 \mathrm{~h}$, the cells were treated with various doses of cisplatin, cultured for another 2 days, and then
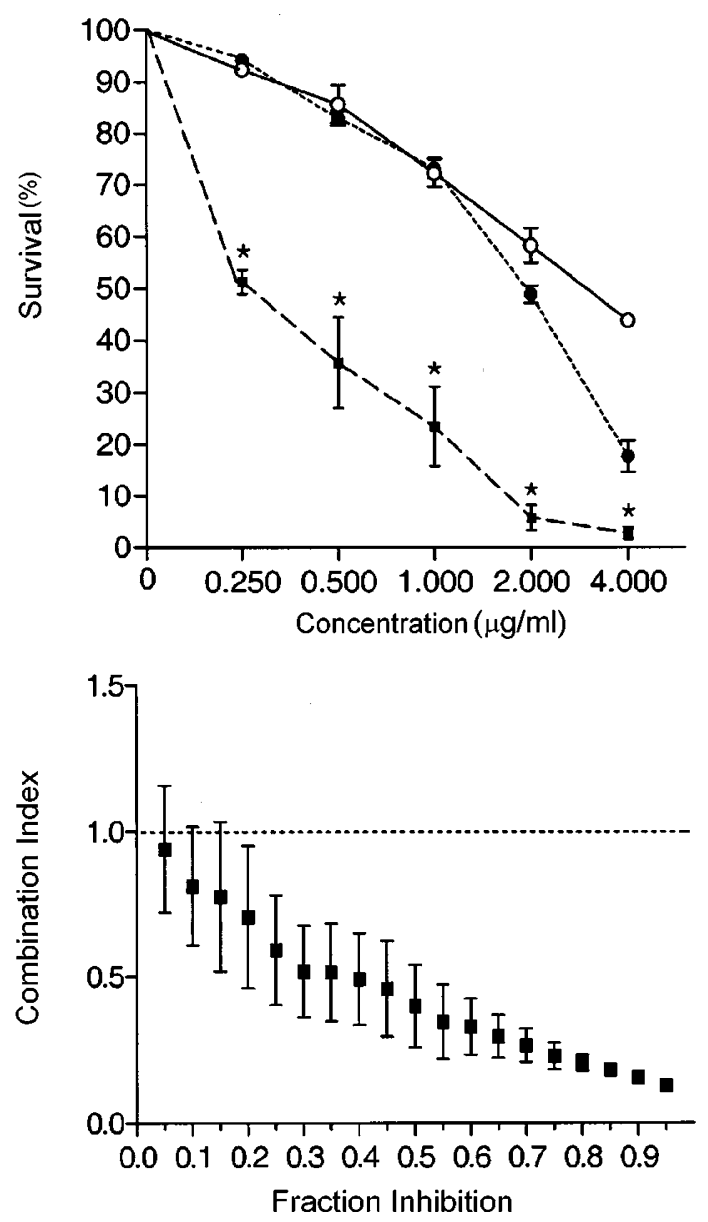

Figure 1. Cisplatin sensitization of SNU638 cells after transfection with an antisense Prx II expression plasmid (pPrxll/AS). Cells were transfected with $0.25,0.5,1,2$, and $4 \mu \mathrm{g} / \mathrm{ml}$ of $\mathrm{pPrxl} / \mathrm{AS}$ respectively, as described in Materials and Methods. The cells were then treated with $0.25,0.5,1,2$, and $4 \mu \mathrm{g} / \mathrm{ml}$ of cisplatin, respectively. After 4 days, cell survival was measured by cell counting and same experiments were repeated three times. Panel A: Survival of cells after treatment with pPrxll/AS $(\bigcirc)$ or cisplatin $(0)$ or pPrxll/AS + cisplatin ( $\mathbf{\square})$. Panel B: Fa-Cl plot. A Cl value $<1$ represents synergy between Prx II antisense and cisplatin. Statistical significance between pPrxll/AS and pPrxll/AS+cisplatin, and between cisplatin and pPrxll/AS + cisplatin was analyzed. ${ }^{*} P<0.01$. harvested. They were stained with propidium iodide and apoptotic cell death was then analyzed by flow cytometric analysis (FACscan; Becton Dickinson, Mountain View, CA), according to the manufacturer's instruction.

\section{Data analysis}

All results are expressed as mean \pm standard deviation (SD). Statistical analysis between each group was performed by one-way analysis of variance (ANOVA). Statistical significance was accepted as $P<0.05$. The combined effect of Prx II antisense and cisplatin was determined by dose-effect analysis, calculated using a microcomputer (Biosoft, Cambridge, England). A combination index $(\mathrm{Cl})$ value $<1$ represents synergy between Prx II antisense and cisplatin, a $\mathrm{Cl}$ value $>1$ indicates antagonism, and a $\mathrm{Cl}$ value of 1 means that the effects of the two agents are additive.

\section{Results}

SNU638 cells were transfected with various concentrations of plasmid, pPrxll/AS, and then treated with the same concentrations of cisplatin. After incubation for 4 days, the cells were counted under a microscope. As shown in Figure 1A, pPrxll/AS itself caused cell death, which is consistent with our previous results on enhanced radiation-induced cell death by pPrxll/AS (Park et al., 2000). Our previous results also demonstrated that pPrxll/AS inhibited Prx II expression (Park et al., 2000; Chung et al., 2001). pPrxll/AS and cisplatin caused $45.1 \%$ and $53.5 \%$ cell death, respectively, at a dose of $2 \mu \mathrm{g}$, when used separately. However, combined treatment with these two agents caused $99.1 \%$ of cell death, suggesting

Table 1. Sequences of Prx II antisense oligomers

\begin{tabular}{cl}
\hline Name & \multicolumn{1}{c}{ Sequence } \\
\hline AS-1 & CAGGTGGGGGCACAGGTGGA \\
AS-2 & TCCGTTAGCCAGCCTAATTG \\
AS-3 & GTTGGGCTTAATCGTGTCAC \\
AS-4 & GTCTGTGTACTGGAAGGCCT \\
AS-5 & CAAATCATTAACAGTGATCT \\
AS-6 & GTAGGCAATGCCCTCATCTG \\
AS-7 & ACGCCGTAATCCTCAGACAA \\
AS-8 & GTCACCAAGCAGGGGGATGT \\
AS-9 & CCAAGCCAGGTGGTTGAACT \\
AS-10 & GCGGAAGTCCTCTGCACGGT \\
AS-11 & GTCCAGAGGGTAGAAAAAGA \\
AS-12 & TTTGAAGGCGCCATCAACCA \\
AS-13 & CGCGCGTTACCGGAGGCCAT \\
AS-14 & AGCTGCGTGGGCAAAGGCTA \\
\hline
\end{tabular}




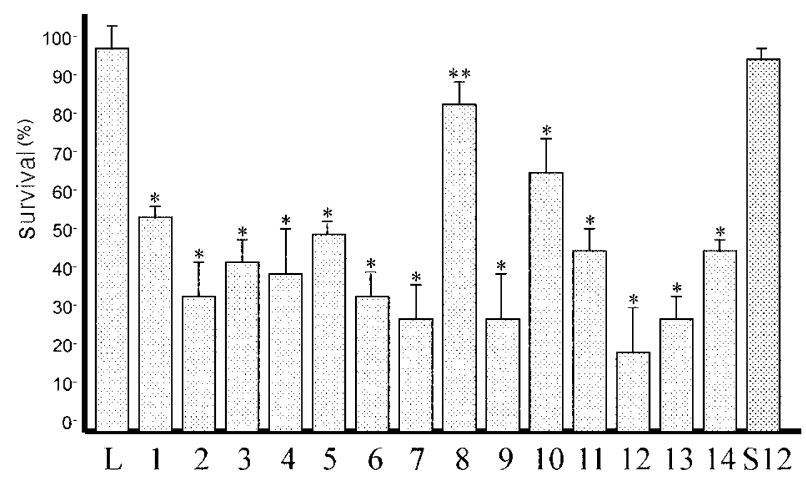

Figure 2. Cell survival in Prx II antisense oligomers-treated SNU638. The cells were transfected twice with each $1 \mathrm{mM}$ antisense oligomers and a sense oligomer as a control. After 4 days, survival curve was determined by cell counting and same experiments were repeated three times. ASs, cells transfected with the corresponding antisense oligomers; S12, cells transfected with S12; L, cells transfected with liposome only. Statistical significance between a sense oligomer (S12) and each antisense oligomers was analyzed. ${ }^{\star} P<0.01,{ }^{\star *} P<0.05$.

that pPrxl//AS enhanced cisplatin-induced cell death. To confirm this synergistic effect, a $\mathrm{Cl}$ plot was drawn. Figure 1B shows the $\mathrm{Cl}$ value below 1 at all doses of the combination, indicating synergism.

In the above experiments, we used the full-length Prx II antisense. In order to examine whether antisense oligomers were also able to sensitize cisplatin-induced cell death, we prepared 14 antisense oligomers, which are listed in Table 1, and carried out similar experiments. Figure 2 shows that all 14 antisense oligomers induced cell death following transfection. Out of the 14 oligomers, we selected the AS12 oligomer as the best antisense oligomer for inducing cell death, and used it for sensitization experiments: One $\mu \mathrm{M}$ of $\mathrm{AS} 12$ induced $80 \%$ cell death. Treatment of the cells with a Prx II sense oligomer (S12), which is complimentary to AS12, did not alter cell viability, compared with the cells transfected with liposome only, thereby demonstrating that Prx II antisense itself was responsible for cell death. On the other hand, when SNU638 cells were transfected with AS12, the higher the dosage of AS12 in the cells, the greater the cell death followed (Figure 3A). When the cells were treated with these two agents together, $82.9 \%$ of cell death was observed, whereas AS12 or cisplatin alone, at a dose of $1.25 \mu \mathrm{g} / \mathrm{ml}$, caused $50.0 \%$ or $42.4 \%$ cell death, respectively, suggesting that the Prx II antisense oligomer also enhanced cisplatin-induced cell death. $\mathrm{A} \mathrm{Cl}$ plot indicated that at all doses of the combination, except for the case of $10 \%$ inhibition, the $\mathrm{Cl}$ was below 1 , thus confirming the synergistic effect (Figure 3B). To demonstrate the effect of Prx II antisense on apoptosis, the cells transfected with $\mathrm{pPrxll} / \mathrm{AS}$, following treatment with cisplatin, was examined by flow cytometry. Figure 4 shows that treatment of the transfected cells with $5 \mu \mathrm{g} / \mathrm{ml}$ of cisplatin for $48 \mathrm{~h}$ induced $32.50 \%$ of apoptosis, whereas $26.13 \%$ of apoptosis was induced in the cells transfected
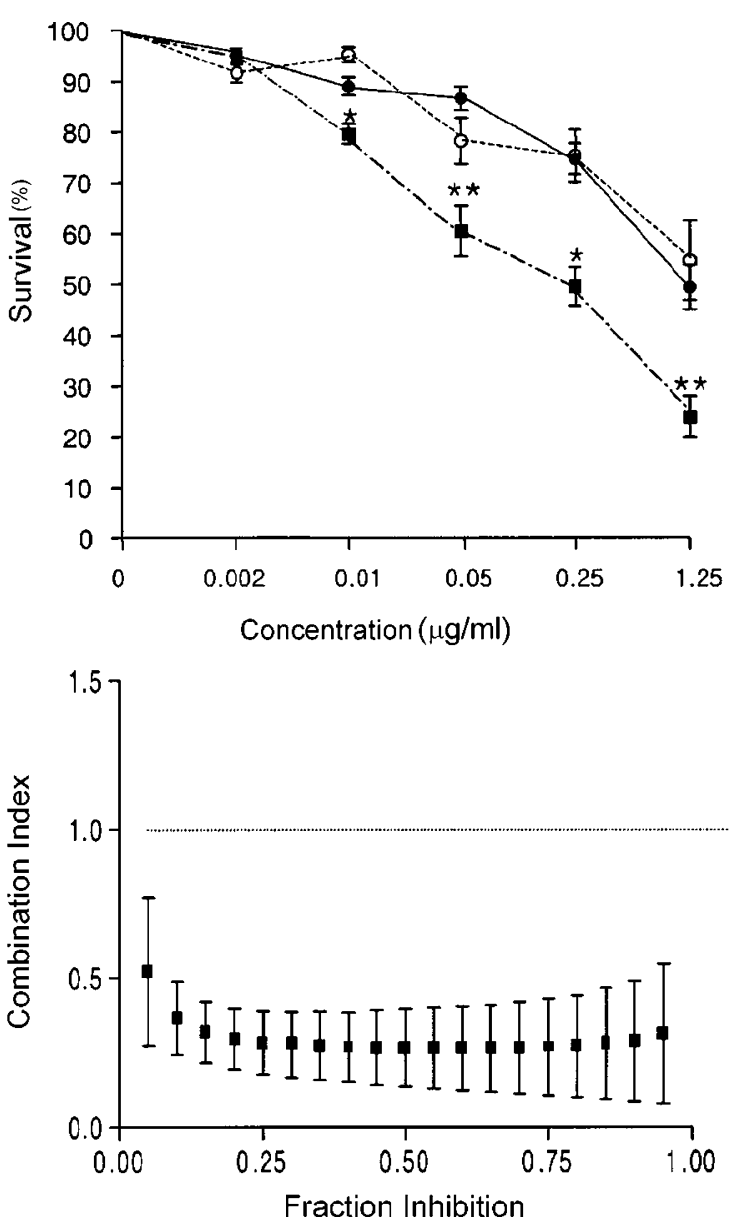

Figure 3. Synergistic effect of an antisense Prx II oligomer, AS-12, combined with cisplatin. Cells were transfected with $0.002,0.01,0.05,0.25$, and $1.25 \mu \mathrm{g} / \mathrm{ml}$ of pPrxll/AS, respectively. The cells were then treated with $0.002,0.01,0.05,0.25$, and $1.25 \mu \mathrm{g} / \mathrm{ml}$ of cisplatin, respectively. After 4 days, cell survival was measured by cell counting and same experiments were repeated three times. Panel A: Survival curve of cells after treatment with pPrxll/AS $(\bigcirc)$ or cisplatin $(-$ ) or pPrxll/AS + cisplatin $(\boldsymbol{\square})$. Panel $\mathrm{B}$ : Fa-Cl plot. Statistical significance between pPrxll/AS and pPrxll/ $\mathrm{AS}+$ cisplatin, and between cisplatin and pPrxll/AS+cisplatin was analyzed. ${ }^{*} P<0.01,{ }^{* *} P<0.05$.

with pPrxll/S. These results suggest that Prx II antisense can be used as a chemosensitizer.

\section{Discussion}

Prx II is an isotype of thioredoxin-dependent peroxidase, which reduces intracellular hydrogen peroxide that are detrimental to cells (Chae et al., 1994). Prx II was known to be induced by exogenous $\mathrm{H}_{2} \mathrm{O}_{2}$ generated by thyrotropin, serum deprivation, ceramide, or etoposide, possibly through eliminating intracellular $\mathrm{H}_{2} \mathrm{O}_{2}$ and other reactive oxygen species (Kim et al., 1997; Zhang et al., 1997; Kim et al., 2000), that are formed as a result of cisplatin treatment or radiation exposure (Sodhi and Gupta, 1986; Park et al., 2000; Leach et al., 2001). We 
A

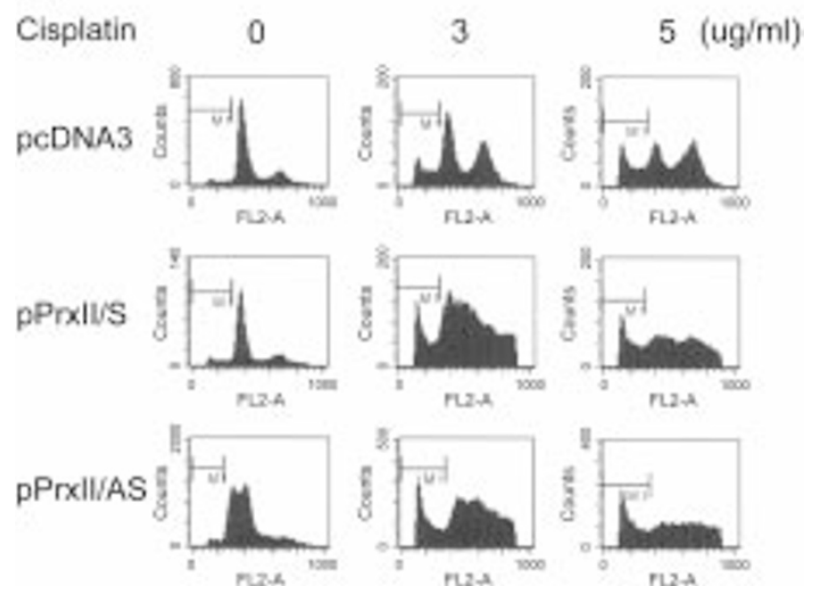

B

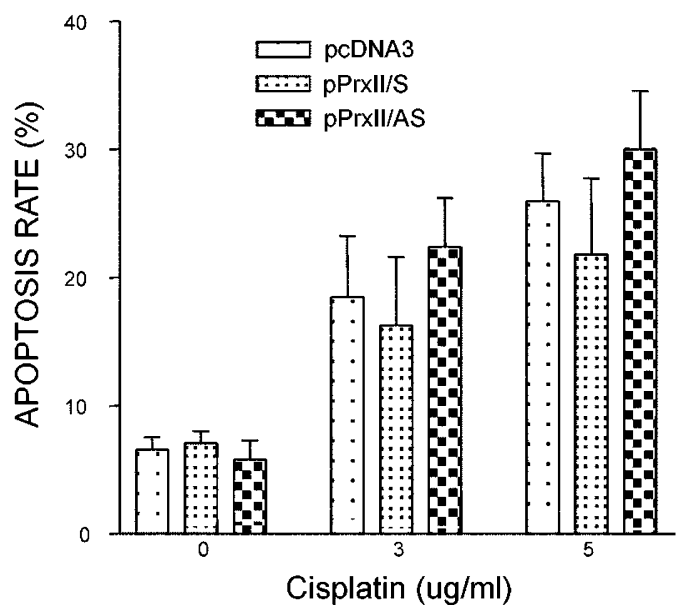

Figure 4. Apoptosis of cells treated with pPrxll/AS and cisplatin. Cells were transfected twice with $0.5 \mu \mathrm{g} / \mathrm{ml}$ of pPrxll/AS. After $16 \mathrm{~h}$, the cells were treated with 3 and $5 \mu \mathrm{g} / \mathrm{ml}$ of cisplatin, respectively. After 2 days, the cells were harvested and stained with propidium iodide, and cell-cycle distribution was then analyzed by flow cytometric analysis. Panel A, cell-cycle distributions; panel $\mathrm{B}$, apoptosis rates of cells after treatment with pPrxll/ $\mathrm{AS}+$ cisplatin or $\mathrm{pPrxll} / \mathrm{S}+$ cisplatin .

earlier showed that increased expression of Prx II was directly related to cisplatin resistance, and suggested that increased activity of Prx II might confer cancer cells with chemoresistance (Chung et al., 2001) or radioresistance (Park et al., 2000). This resistance seemed to be derived from the presence of an increased amount of Prx II, enabling the elimination of hydrogen peroxide produced as a consequence of cisplatin or radiation treatment. Cells resistant to $\mathrm{H}_{2} \mathrm{O}_{2}$ were reported to be resistant to cisplatin (Spitz et al., 1993), and down-regulation by Prx II antisense sensitized radiation-induced cell death (Park et al., 2000). Indeed, as shown in the present results, Prx II antisense effectively enhanced cisplatin-induced cell death. Even though, in this report, we showed only that Prx II anti-sense sensitized cisplatin-induced cell death, we suggest that Prx II antisense could also enhance other instances of anticancer drug-induced cell death, especially in the case of anticancer drugs which generate reactive oxy-gen species. This observation is consistent with pre-vious results (Zhang et al., 1997) where Prx II overexpression inhibited apoptosis in Molt4 leukemia cells and functioned in a similar manner to Bcl-2. Also, Prx II overexpression rendered gastric cancer and ECV304 cells more resistant to chemotherapeutic drugs (Kang et al., 1998).

Cellular resistance to anticancer drugs and $\gamma$-radiation is one of the difficulties encountered in the effective treatment of numerous cancers. Therefore, the enhancement of cellular sensitivity toward chemo- or radiation therapy constitutes an important field of research for improving cancer treatments. Because blocking the expression of Prx II disrupts total cellular redox homeostasis and induces apoptosis (Sato et al., 1995), Prx II could be the focus of new drugs for use in the treatment of cancer. Our data, presented herein, strongly suggest that inactivation of the stress-activated protein Prx II constitutes a promising approach to the development of improved cancer treatments, and that inhibitors of Prx II represent very good anticancer drug candidates, especially in the role of chemosensitizers or radio-sensitizers.

\section{Acknowledgements}

This study was supported by a grant, No. R01-200000118, from the Basic Research Program of the Korea Science and Engineering Foundation.

\section{References}

Butterfield LH, Merino A, Golb SH, Shau H. From cytoprotection to tumor suppression: the multifactorial role of peroxiredoxins. Antiox Redox Signal 1999;1:385-402

Chae HZ, Chung SJ, Rhee SG. Thioredoxin-dependent Peroxide Reductase from Yeast. J Biol Chem 1994;269: 27670-8

Chae HZ, Kang SW, Rhee SG. Isoforms of Mammalian Peroxiredoxin That Reduce Peroxides in Presence of Thioredoxin. Methods Enzymol 1999;300:219-6

Chae HZ, Kim HJ, Kang SW, Rhee SG. Characterization of three isoforms of mammalian peroxiredoxin that reduce peroxides in the presence of thioredoxin. Diabetes Res Clin Pract 1999;45:101-2

Chang JW, Jeon HB, Lee JH, Yoo JS, Chun JS, Kim JH, Yoo YJ. Augmented expression of peroxiredoxin I in lung cancer. Biochem Biophys Res Commun 2001;289:507-12

Chung YM, Yoo YD, Park JK, Kim YT, Kim HJ. Increased expression of peroxiredoxin II confers resistance to cisplatin. Anticancer Res 2001;21:1129-34

Kang SW, Chae HZ, Seo MS, Kim K, Baines IC, Rhee SG. 
Mammalian Peroxiredoxin Isoforms Can Reduce Hydrogen Peroxide Generated in Response to Growth Factors and Tumor Necrosis Facor- $\alpha$. J Biol Chem 1998;273:6297-302

Kim AT, Sarafian TA, Shau H. Characterization of antioxidant properties of natural killer-enhancing factor-B and induction of its expression by hydrogen peroxide. Toxicol Appl Pharmacol 1997; 147:135-42

Kim H, Lee TH, Park ES, Suh JM, Park SJ, Chung HK, Kwon OY, Kim YK, Ro HK, Shong M. Role of peroxiredoxins in regulating intracellular hydrogen peroxide and hydrogen peroxide-induced apoptosis in thyroid cells. J Biol Chem 2000;275:18266-70

Leach JK, Van Tuyle G, Lin PS, Schmidt-Ullrich R, Mikkelsen RB. lonizing radiation-induced, mitochondria-dependent generation of reactive oxygen/nitrogen. Cancer Res 2001;61:3894-901

Noh DY, Ahn SJ, Lee RA, Kim SW, Park IA, Chae HZ. Overexpression of peroxiredoxin in human breast cancer: Anticancer Res 2001;21:2085-90

Park SH, Chung YM, Lee YS, Kim HJ, Kim JS, Chae HZ, Yoo YD. Antisense of human peroxiredoxin II enhances radiationinduced cell death. Clin Cancer Res 2000;6:4915-20

Sato N, Iwata S, Nakamura K, Hori T, Mori K, Yodoi J. Thiolmediated redox regulation of apoptosis. Possible role of cellular thiols other than glutathione in $\mathrm{T}$ cell apoptosis. $\mathrm{J}$
Immunol 1995;154:3194-203

Sen CK. Redox signaling and the emerging therapeutic potential of thiol antioxidants. Biochem Pharmacol 1998;55: 1747-58

Sodhi A, Gupta P. Increased release of hydrogen peroxide $\left(\mathrm{H}_{2} \mathrm{O}_{2}\right)$ and superoxide anion $\left(\mathrm{O}_{2}^{-}\right)$by murine macrophages in vitro after cis-platin treatment. Int $\mathrm{J}$ Immunopharmacol 1986;8:709-14

Spitz DR, Phillips JW, Adams DT, Sherman CM, Deen DF, Li GC. Cellular resistance to oxidative stress is accompanied by resistance to cisplatin: the significance of increased catalase activity and total glutathione in hydrogen peroxide-resistant fibroblasts. J Cell Physiol 1993;156:72-9

Yanagawa T, Ishikawa T, Ishii T, Tabuchi K, Iwasa S, Bannai S, Omura K, Suzuki H, Yoshida H. Peroxiredoxin I expression in human thyroid tumors. Cancer Lett 1999;145:127-32

Yanagawa T, Iwasa S, Ishii T, Tabuchi K, Yusa H, Onizawa K, Omura K, Harada H, Suzuki H, Yoshida H. Peroxiredoxin I expression in oral cancer: a potential new tumor marker. Cancer Lett 2000;156:27-35

Zhang P, Liu B, Kang SW, Seo MS, Rhee SG, Obeid LM. Thioredoxin peroxidase is a novel inhibitor of apoptosis with a mechanism distinct from that of Bcl-2. J Biol Chem 1997;272: 30615-8 